\title{
Casimir interaction between two smoothly deformed cylindrical surfaces
}

\author{
J. D. Melon Fuksman ${ }^{1,2, *}$ and C. D. Fosco ${ }^{3}$ \\ ${ }^{1}$ Dipartimento di Fisica, Sapienza Università di Roma, Piazzale Aldo Moro 5, 00185 Rome, Italy \\ ${ }^{2}$ ICRANet, Piazza della Repubblica 10, 65122 Pescara, Italy \\ ${ }^{3}$ Centro Atómico Bariloche and Instituto Balseiro, Comisión Nacional de Energía Atómica 8400 \\ Bariloche, Argentina
}

(Received 25 July 2017; published 25 October 2017)

\begin{abstract}
We generalize the derivative expansion (DE) approach to the interaction between almost-flat smooth surfaces, to the case of surfaces which are optimally described in cylindrical coordinates. As in the original form of the DE, the obtained method does not depend on the nature of the interaction. We apply our results to the study of the static, zero-temperature Casimir effect between two cylindrical surfaces, obtaining approximate expressions which are reliable under the assumption that the distance between those surfaces is always much smaller than their local curvature radii. To obtain the zero-point energy, we apply known results about the thermal Casimir effect for a planar geometry. To that effect, we relate the time coordinate in the latter to the angular variable in the cylindrical case, as well as the temperature to the radius of the cylinders. We study the dependence of the applicability of the DE on the kind of interaction, considering the particular cases where Dirichlet or Neumann conditions are applied to a scalar field.
\end{abstract}

DOI: 10.1103/PhysRevD.96.076015

\section{INTRODUCTION}

The Casimir effect has been justly regarded as one of the most startling macroscopic manifestations of fluctuations, be them quantum mechanical or thermal, of a field [1].

To make predictions about the Casimir effect, typically involves evaluating the influence of nontrivial boundary conditions on the vacuum (or thermal) expectation values of the relevant observables. That task is, except when highly symmetrical geometries are considered, rather involved. One of the main reasons for that is that those expectation values usually do not satisfy a superposition principle, when regarded as functionals of the boundary. Thus, it is not possible, in general, to calculate the total energy in the presence of a given boundary, by adding the contributions due to each one of the possible pairs of surface elements into which the boundary may be decomposed [2]. As a consequence, rather few "universal" (i.e., applicable to an arbitrary surface) properties of the Casimir effect are known.

The motivation to develop approximate methods to deal with rather general geometries hardly needs to be emphasised. One of those methods, of much wider applicability than the Casimir effect, is the so called proximity force approximation (PFA), originally introduced by B. Derjaguin in 1934 [3], within the context of the interaction between interfaces. This method has subsequently been applied to several unrelated areas, like nuclear physics [4,5], Van der Waals interactions and, lately, the Casimir effect [1], with varying degree of success.

In its most frequently used version, the PFA is applied to a setup consisting of two interacting surfaces, $L$ and $R$, such

*david.melon@icranet.org that $L$ is assumed to be a plane, and $R$, which (also by assumption) can be represented by means of a single function, $\psi\left(\mathbf{x}_{\|}\right)$, the height of $R$ at each point $\mathbf{x}_{\|}$of $L$.

Then, $E_{\mathrm{PFA}}$, the PFA approximation to the interaction energy $E$ between the surfaces, is:

$$
E_{\mathrm{PFA}} \equiv \int d \sigma \mathcal{E}_{\|}\left[\psi\left(\mathbf{x}_{\|}\right)\right]
$$

where $d \sigma$ is the area element at a point $\mathbf{x}_{\|}$on $L$, and $\mathcal{E}_{\|}(h)$ denotes the energy per unit area for two parallel surfaces, i.e., for $\psi\left(\mathbf{x}_{\|}\right) \equiv h$, where $h$ is a constant. ${ }^{1}$

Until quite recently, there were no known controlled ways of generalizing the PFA, so as to include shapedependent corrections in an ordered perturbative expansion. A step in that direction has been taken with the introduction of the derivative expansion (DE) [6-10], an approach that leads to a modification of (1) whereby the surface energy density function includes derivatives of $\psi$, meant to account for a dependence on the surface's local curvature. Successive terms in the expansion have an increasing numbers of derivatives of $\psi$; the PFA being reinterpreted as the zeroth (leading) order term in that expansion.

This kind of approach is quite independent of the nature of the interaction, what makes its potential range of applicability rather wide. However, the implementation of the DE for surfaces that cannot be described by using a single Monge patch is problematic, in part because of the

\footnotetext{
${ }^{1}$ The approximation above can be generalized to two curved surfaces whenever they may be both represented by two functions, $\psi_{L}, \psi_{R}$, which measure the respective height about a common reference surface.
} 
seemingly important role played by the Fourier transformation of the distance function $\psi$, when written in Cartesian coordinates [11], and also because of the different topology of the manifolds. This article presents an answer to that point, for the specific case of two cylindrical surfaces. Besides implementing the DE, we also show that the functions that define it may be derived from known results obtained for a planar system at a finite temperature [10], by relating the periodicity of the imaginary time to the one of the angular variable.

Next-to-leading order corrections to the PFA have already been calculated for particular cases of almostcylindrical surfaces, for instance, in $[12,13]$. This kind of geometry is also interesting from the experimental point of view, since it can be used to create configurations that allow us to measure lateral Casimir forces, as it is analyzed in these references.

This paper is organized as follows: in Sec. II, after briefly reviewing the DE in its usual, single Monge patch formulation in II A, we present in II B the analogous construction for cylindrical surfaces. Technical details of the derivation are presented in an Appendix.

In Sec. III, we apply the DE to the Casimir energy for a quantum real scalar field satisfying either Dirichlet or Neumann conditions. Finally, in Sec. IV, we summarize our conclusions.

\section{THE DERIVATIVE EXPANSION}

\section{A. Standard formularion}

We begin by reviewing the main features of the DE in its simplest setup: two surfaces, $L$ and $R$, as the ones mentioned in the previous Section. More specifically, we assume that a Cartesian coordinate system has been chosen such that $L$ and $R$ occupy the regions (subsets of $\mathbb{R}^{3}$ ) given by: $s_{L} \equiv\left\{\left(x_{1}, x_{2}, 0\right)\right\}$ and $s_{R}=\left\{\left(x_{1}, x_{2}, \psi\left(\mathbf{x}_{\|}\right)\right)\right\}$, respectively. Here, $\mathbf{x}_{\|} \equiv\left(x_{1}, x_{2}\right)$ and $\psi$ is a smooth function of $\mathbf{x}_{\|}$. Let $F[\psi]$ denote the interaction energy between the two surfaces (not necessarily originated in the Casimir effect) written as a functional of $\psi$. The DE yields an approximation to $F$ as a series of local terms, ordered according to their increasing number of derivatives of $\psi$. Up to the second order $^{2}$ :

$$
F[\psi]=F_{0}[\psi]+F_{2}[\psi]+\cdots
$$

with

$F_{0}[\psi]=\int_{\mathbf{x}_{\|}} V\left(\psi\left(\mathbf{x}_{\|}\right)\right), \quad F_{2}[\psi]=\int_{\mathbf{x}_{\|}} Z\left(\psi\left(\mathbf{x}_{\|}\right)\right)|\nabla \psi|^{2}$

(see, for example, [11]).

\footnotetext{
${ }^{2}$ Although in principle one could consider an arbitrary number of orders, the number of terms involved and the complexity involved in their calculation grows rather fast.
}

Once the functions $V$ and $Z$ are determined, by any suitable method, the previous equations may then be used to obtain approximate values for the interaction energy between surfaces having different geometries, namely, defined by different functions $\psi$.

One can see that the zeroth order term $F_{0}[\psi]$ above does reduce to the PFA. Indeed, considering a (temporarily) finite integration area $S$, and a constant $\psi\left(\mathbf{x}_{\|}\right)=a$, all the terms but the first one vanish. Hence, the function $V$ may be determined as follows:

$$
V(a)=\lim _{S \rightarrow \infty}\left(\frac{F[a]}{S}\right) .
$$

Thus,

$$
F_{0}[\psi]=\int_{\mathbf{x}_{\|}} V\left(\psi\left(\mathbf{x}_{\|}\right)\right),
$$

which agrees, mutatis mutandis with the PFA (1).

The next-to-leading-order (NTLO) term $F_{2}$, is in turn determined by the $Z$ function, which may be obtained in an analogous way. For, example, one can evaluate $F[\psi]$ for $\psi\left(\mathbf{x}_{\|}\right)=a+\eta\left(\mathbf{x}_{\|}\right)$, where $\eta$ is a function of $\mathbf{x}_{\|}$, whose mean value is 0 , while $a$ is the average distance between the two surfaces. Expanding $F$ up to the second order in $\eta, Z$ may be extracted from the second order term in a momentum expansion of the Fourier transform of $F$ [11].

\section{B. DE for cylindrical surfaces}

We present here the conventions and results about the DE, when applied to cylindrical surfaces, assuming their geometries may be naturally described in terms of cylindrical coordinates $(\rho, \varphi, z)$. Details regarding the derivation of this result are presented in the Appendix.

The geometry corresponds again to two surfaces, which we now denote by $I$ and $O$. We retain the property that one of them is a coordinate surface, and the other can be defined by giving the distance of each one of its points to the first one. Indeed, we assume now $s_{I}$ to be a constant- $\rho$ coordinate surface, namely, a circular cylinder of radius $\rho=a$, while $R$ occupies a region $s_{O}$ such that, for any given value of $\varphi$ and $z$, its radius is determined by a single function $\psi: \rho=\psi(\varphi, z)$. As in the previous subsection, we decompose $\psi$ into its average and its departure about it: $\psi(\varphi, z)=b+\eta(\varphi, z)$.

The procedure outlined in the Appendix shows that the $\mathrm{DE}$, up to the second order, is given by the expression:

$$
F[\psi]=F_{0}[\psi]+F_{2}[\psi]+\cdots
$$

where

$$
F_{0}[\psi]=\int_{x} b \mathcal{F}_{0}(b+\eta(x))
$$


and

$$
\begin{aligned}
F_{2}[\psi]= & \int_{x}\left\{Z_{1}(\psi(x))\left(\partial_{z} \psi\right)^{2}+Z_{2}(\psi(x))\left(\partial_{\varphi} \psi\right)^{2}\right. \\
& \left.+Z_{12}(\psi(x))\left(\partial_{\varphi} \psi\right)\left(\partial_{z} \psi\right)\right\} .
\end{aligned}
$$

This result relies upon the same assumptions as the Cartesian case, except for an extra condition, namely, that $\psi$ and its derivatives are periodic functions of the angular variable. Besides, we have kept a mixed term involving derivatives with respect to the angle and $z$. The reason to keep that term is that one might want to apply the approximation to systems where an external field breaks, for example, the invariance under $\varphi \rightarrow-\varphi$. One may imagine, for example, the existence of an external magnetic field along $z$. When there are sufficient symmetries, that term will of course vanish.

\section{APPLICATIONS}

In this section, we apply the DE for cylindrical surfaces to the interaction energy resulting from the Casimir energy for a real scalar field. We work within the functional integral approach, in the imaginary time formulation, where the spacetime metric becomes the identity matrix when Cartesian coordinates $x=\left(x_{0}, x_{1}, x_{2}, x_{3}\right)$ are adopted, $x_{0}$ denoting the Euclidean (imaginary) time. Spatial coordinates are denoted collectively by $\mathbf{x}$.

The vacuum energy, which we shall denote by $E_{0}$, may be obtained as the zero-temperature limit of the free energy (see, e.g., [14]), by means of the expression

$$
E_{0}=-\lim _{\beta \rightarrow \infty} \beta^{-1} \log \mathcal{Z},
$$

where $\mathcal{Z}$ denotes the canonical partition function for a temperature $T=\beta^{-1}$ (natural units where $k_{B}=1$ have been adopted). This expression must not be mistaken for the energy of the free vacuum, since boundary conditions may-and will—be included in $\mathcal{Z}$.

To include such boundary conditions, we will use two $\delta$-functionals: $\delta_{I}$ and $\delta_{O}$, respectively. $\mathcal{Z}$, which will be a functional of $\psi$, can then be written as follows:

$$
\mathcal{Z}[\psi]=\int \mathcal{D} \phi \delta_{I}[\phi] \delta_{O}[\phi] e^{-\mathcal{S}_{0}[\phi]},
$$

where the integral is over $\phi$ configurations which are periodic in the time interval $\left[-\frac{\beta}{2}, \frac{\beta}{2}\right]$, and $\mathcal{S}_{0}[\phi]$ is the free Euclidean action. This shall be given by:

$$
\mathcal{S}_{0}[\phi]=\frac{1}{2} \int d^{4} x(\partial \phi)^{2},
$$

where the $x_{0}$ integral runs from $-\frac{\beta}{2}$ to $+\frac{\beta}{2}$. Similarly, we define $\mathcal{Z}_{0}$ as the partition function when no boundary conditions are applied.

\section{A. Scalar field with Dirichlet conditions}

We first consider a real scalar field $\phi$ and approximately cylindrical surfaces, upon which Dirichlet conditions are imposed. The world volumes swept by those surfaces will be parametrized as $s_{I}=\left\{\left(x_{0}, \rho \cos \varphi, \rho \sin \varphi, z\right): \rho=a\right\}$ and $s_{O}=\left\{\left(x_{0}, \rho \cos \varphi, \rho \sin \varphi, z\right): \rho=\psi(\varphi, z)\right\}$. Here, $\varphi \in[-\pi, \pi)$, $x_{0} \in(-\infty, \infty)$, and $z \in(-\infty, \infty)$.

We assume that $\psi(\varphi, z)=b+\eta(\varphi, z)$, with $b>a$, and $\eta$ a differentiable function such that $|\eta(\varphi, z)| \ll b-a$, $\forall \varphi, z$. Following the derivation in the Appendix, we choose $b$ so that

$$
\int_{-\pi}^{\pi} \mathrm{d} \varphi \int_{-\infty}^{\infty} \mathrm{d} z \eta(\varphi, z)=0
$$

and hence the surface $s_{O}$ is, on average, a cylinder of radius $b$, and the first order term in the functional expansion in powers of $\eta$, vanishes.

To impose the condition $\phi_{s_{I}, s_{O}}=0$, we insert in $\mathcal{Z}[\psi]$ the functionals $\delta_{I}[\phi]$ and $\delta_{O}[\phi]$, defined in terms of auxiliary fields $\xi_{I}(y)$ and $\xi_{O}(y)$ as:

$\delta_{I}[\phi]=\int \mathcal{D} \xi_{I} \exp \left[i \int_{y} \xi_{I}(y) \phi(y) \delta(\rho-a)\right]$

$\delta_{O}[\phi]=\int \mathcal{D} \xi_{O} \exp \left[i \int_{y} \sqrt{g\left(y_{\|}\right)} \xi_{O}(y) \phi(y) \frac{\delta\left(\rho-\psi\left(y_{\|}\right)\right)}{\rho}\right]$

where $y \equiv\left(x_{0}, \rho, \varphi, z\right), y_{\|} \equiv\left(x_{0}, \varphi, z\right), \int_{y} \equiv \int_{-\pi}^{\pi} \mathrm{d} \varphi \int_{-\infty}^{\infty} \mathrm{d} z \times$ $\int_{-\infty}^{\infty} \mathrm{d} x_{0} \int_{0}^{\infty} \rho \mathrm{d} \rho$ and $g\left(y_{\|}\right)$is the determinant of the metric induced on $s_{O}$.

Integrating out $\phi$, we see that:

$\frac{\mathcal{Z}[\psi]}{\mathcal{Z}_{0}}=\int \mathcal{D} \xi_{I} \mathcal{D} \xi_{O} \exp \left[-\frac{1}{2} \int_{y_{\|}, y_{\|}^{\prime}} \xi_{A}\left(y_{\|}\right) T_{A B}\left(y_{\|}, y_{\|}^{\prime}\right) \xi_{B}\left(y_{\|}^{\prime}\right)\right]$,

where $A$ and $B$ may be $I$ or $O$, and $T_{A B}\left(y_{\|}, y_{\|}^{\prime}\right)$ are the components of a matrix kernel $\mathbb{T}$, defined as $\mathbb{T}\left(y_{\|}, y_{\|}^{\prime}\right)=$ $\mathbb{M}\left(y_{\|}\right) \mathbb{D}\left(y_{\|}, y_{\|}^{\prime}\right) \mathbb{M}\left(y_{\|}^{\prime}\right)$, with

$$
\begin{aligned}
\mathbb{M}\left(y_{\|}\right) & =\left(\begin{array}{cc}
a & 0 \\
0 & \sqrt{g\left(y_{\|}\right)}
\end{array}\right) \\
& =\left(\begin{array}{lc}
a & 0 \\
0 & \sqrt{\left(\partial_{\phi} \psi\right)^{2}+\psi^{2}\left(1+\left(\partial_{z} \psi\right)^{2}\right)}
\end{array}\right),
\end{aligned}
$$

and $\mathbb{D}\left(y_{\|}, y_{\|}^{\prime}\right)=\left(\begin{array}{cc}D_{I I} & D_{I O} \\ D_{O I} & D_{O O}\end{array}\right)$. The latter are 


$$
\begin{aligned}
D_{I I}\left(y_{\|}, y_{\|}^{\prime}\right) & =\left\langle y_{\|}, a\left|\left(-\partial^{2}\right)^{-1}\right| y_{\|}^{\prime}, a\right\rangle \\
D_{I O}\left(y_{\|}, y_{\|}^{\prime}\right) & =\left\langle y_{\|}, a\left|\left(-\partial^{2}\right)^{-1}\right| y_{\|}^{\prime}, \psi\left(\varphi^{\prime}, z^{\prime}\right)\right\rangle \\
D_{O I}\left(y_{\|}, y_{\|}^{\prime}\right) & =\left\langle y_{\|}, \psi(\varphi, z)\left|\left(-\partial^{2}\right)^{-1}\right| y_{\|}^{\prime}, a\right\rangle \\
D_{O O}\left(y_{\|}, y_{\|}^{\prime}\right) & =\left\langle y_{\|}, \psi(\varphi, z)\left|\left(-\partial^{2}\right)^{-1}\right| y_{\|}^{\prime}, \psi\left(\varphi^{\prime}, z^{\prime}\right)\right\rangle
\end{aligned}
$$

where $\left\langle y_{\|}, \rho\left|\left(-\partial^{2}\right)^{-1}\right| y_{\|}^{\prime}, \rho^{\prime}\right\rangle$ is the (free) $\phi$-field propagator.

Thus, neglecting irrelevant contributions:

$$
E_{0}=\lim _{\beta \rightarrow \infty} \frac{1}{2 \beta} \operatorname{Tr} \log \mathbb{T} .
$$

In the following subsections, we consider the different terms in the expansion of (18) in powers of $\eta$ which are needed to construct the DE.

\section{0th-order term}

To this order, we need to take $\psi \equiv b$, and find the resulting matrix elements $\mathbb{T}$. These may be obtained using the propagator definition explicitly, writing the momenta $\mathbf{k}_{\|}=\left(k_{1}, k_{2}\right)$ in polar coordinates, and using the identities

$$
e^{i x \cos \alpha}=\sum_{m=-\infty}^{\infty} i^{m} J_{m}(x) e^{m \alpha}
$$

and

$$
\int_{0}^{\infty} \mathrm{d} s \frac{s}{\mathbf{k}_{\|}^{2}+s^{2}} J_{n}^{2}(s a)=I_{n}\left(\left|\mathbf{k}_{\|}\right| a\right) K_{n}\left(\left|\mathbf{k}_{\|}\right| a\right),
$$

which is valid for every $n \in \mathbb{Z}$. In these expressions, $J_{n}$ are Bessel functions of order $n$, while $I_{n}$ and $K_{n}$ denote modified Bessel functions. This leads to the result:

$\mathbb{T}\left(y_{\|}, y_{\|}^{\prime}\right)=\int_{\mathbf{k}_{\|}} e^{i \mathbf{k}_{\|}\left(\mathbf{y}_{\|}-\mathbf{y}_{\|}^{\prime}\right)} \frac{1}{2 \pi} \sum_{n} e^{i n\left(\varphi-\varphi^{\prime}\right)} \tilde{\mathbb{T}}\left(\mathbf{k}_{\|}, n\right)$,

with

$$
\begin{aligned}
& \tilde{\mathbb{T}}\left(\mathbf{k}_{\|}, n\right) \\
& \quad=\left(\begin{array}{cc}
a^{2} I_{n}\left(\left|\mathbf{k}_{\|}\right| a\right) K_{n}\left(\left|\mathbf{k}_{\|}\right| a\right) & a b I_{n}\left(\left|\mathbf{k}_{\|}\right| a\right) K_{n}\left(\left|\mathbf{k}_{\|}\right| b\right) \\
a b I_{n}\left(\left|\mathbf{k}_{\|}\right| a\right) K_{n}\left(\left|\mathbf{k}_{\|}\right| b\right) & b^{2} I_{n}\left(\left|\mathbf{k}_{\|}\right| b\right) K_{n}\left(\left|\mathbf{k}_{\|}\right| b\right)
\end{array}\right),
\end{aligned}
$$

and $\mathbf{y}_{\|} \equiv\left(x_{0}, z\right)$.

Thus, the interaction energy per unit length $\mathcal{E}_{0}^{l}$ becomes:

$$
\begin{aligned}
\mathcal{E}_{0}^{l} & =\lim _{\beta \rightarrow \infty, L \rightarrow \infty} \frac{1}{2 \beta L} \operatorname{Tr} \log \mathbb{T} \\
& =\frac{1}{2} \int_{\mathbf{k}_{\|}} \sum_{n} \log \operatorname{det} \tilde{\mathbb{T}}\left(\mathbf{k}_{\|}, n\right) .
\end{aligned}
$$

Evaluating the determinant, and discarding contributions which represent self-energy terms (i.e., depending on each separate surface), we arrive to the result:

$$
\mathcal{E}_{0}^{l}=\frac{1}{2} \int_{\mathbf{k}_{\|}} \sum_{n} \log \left[1-\frac{I_{n}\left(\left|\mathbf{k}_{\|}\right| a\right) K_{n}\left(\left|\mathbf{k}_{\|}\right| b\right)}{I_{n}\left(\left|\mathbf{k}_{\|}\right| b\right) K_{n}\left(\left|\mathbf{k}_{\|}\right| a\right)}\right],
$$

which is valid for any $a<b$. This agrees with the known result for this case [15].

We know that the energy per unit area corresponding to the above result should approach the analogous result for a couple of parallel planes when the cylinders are sufficiently close to each other. Let us study this now, deriving at an intermediate step an approximate expression, which is neither the result for cylinders nor for planes: it will correspond to planes with a periodic coordinate, related to the angular variable. When $d \equiv b-a \ll a$, we can use the $n \rightarrow \infty$ approximations $[16,17]$ :

$$
I_{n}(n z) \approx \sqrt{\frac{t}{2 \pi n}} e^{n \xi(z)}
$$

and

$$
K_{n}(n z) \approx \sqrt{\frac{\pi t}{2 n}} e^{-n \xi(z)}
$$

where $t=\frac{1}{\sqrt{1+z^{2}}}$ and $\xi(z)=\sqrt{1+z^{2}}+\log \left(\frac{z}{1+\sqrt{1+z^{2}}}\right)$.

Thus, when $d \ll a$, the ratio in (24) can be approximated as follows

$$
\begin{aligned}
& \frac{I_{n}\left(\left|\mathbf{k}_{\|}\right| a\right) K_{n}\left(\left|\mathbf{k}_{\|}\right| b\right)}{I_{n}\left(\left|\mathbf{k}_{\|}\right| b\right) K_{n}\left(\left|\mathbf{k}_{\|}\right| a\right)} \approx e^{2 n\left[\xi\left(z_{1}\right)-\xi\left(z_{2}\right)\right]}
\end{aligned}
$$

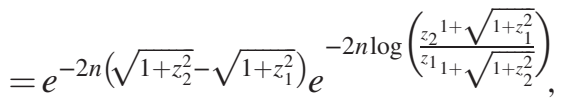

with $z_{1}=\left|\mathbf{k}_{\|}\right| a / n$ and $z_{2}=\left|\mathbf{k}_{\|}\right| b / n$. Next, expanding the exponents in (27) for $d \ll a$, we have found that the most accurate way to do so is to write the result in terms of $d$ and $r=(b+a) / 2$, obtaining:

$$
\frac{I_{n}\left(\left|\mathbf{k}_{\|}\right| a\right) K_{n}\left(\left|\mathbf{k}_{\|}\right| b\right)}{I_{n}\left(\left|\mathbf{k}_{\|}\right| b\right) K_{n}\left(\left|\mathbf{k}_{\|}\right| a\right)} \approx e^{-2 d \sqrt{(n / r)^{2}+\mathbf{k}_{\|}^{2}}} .
$$

Therefore,

$$
\mathcal{E}_{0}^{l} \approx \frac{1}{2} \int_{\mathbf{k}_{\|}} \sum_{n} \log \left(1-e^{-2 d \sqrt{(n / r)^{2}+\mathbf{k}_{\|}^{2}}}\right),
$$

which is the intermediate expression mentioned above; indeed, it contains a sum over a discrete "momentum," 
corresponding to the angular variable. This expression, when divided by $2 \pi r$, yields the energy per unit area $\mathcal{E}_{0}(r)$. Moreover, it tends to the proper limit, i.e., to the parallel planes result when $r \rightarrow \infty$ :

$$
\mathcal{E}_{0}(\infty)=\lim _{r \rightarrow \infty} \mathcal{E}_{0}(r)=\frac{1}{2} \int_{k_{\|}} \log \left(1-e^{-2 d\left|k_{\|}\right|}\right) .
$$

The way in which the limit is reached, may be studied by considering the difference between these two magnitudes,

$$
\begin{aligned}
\mathcal{E}_{0}(r)-\mathcal{E}_{0}(\infty) \\
\quad=2 r \int_{-\infty}^{\infty} \frac{1}{2 \pi} \int \mathrm{d} t \int_{\left(k_{0}, k_{1}\right)} \frac{\log \left(1-e^{-2 d \sqrt{k_{0}^{2}+k_{1}^{2}-(\epsilon+i t)^{2}}}\right)}{e^{2 \pi r(\epsilon+i t)}-1},
\end{aligned}
$$

(where we converted the series to an integral), which is not analytic at $r \rightarrow \infty$, since $e^{\alpha x}$ has an essential singularity at $x \rightarrow \infty$. Namely, it is not possible to expand $\mathcal{E}_{0}(r)-\mathcal{E}_{0}(\infty)$ as a series in powers of $1 / r$.

\section{2nd order}

To obtain the function $Z_{2}$ in the DE, we evaluate in this subsection the second order term in the energy. We do this by applying the analogy between the cylindrical geometry and a planar system at a finite temperature, in the limit $d \ll r$. The approximation will be checked in the concrete example for which the exact result is known, namely, that of eccentric cylinders.

To perform this comparison, we consider the partition functions $\mathcal{Z}^{P}[\psi]$ for approximately flat surfaces described in Cartesian coordinates, and $\mathcal{Z}^{C}[\psi]$, for cylindrical surfaces:

$$
\begin{aligned}
& \frac{\mathcal{Z}^{P}[\psi]}{\mathcal{Z}_{0}^{P}} \\
& \quad=\int \mathcal{D} \xi_{L} \mathcal{D} \xi_{R} \exp \left[-\frac{1}{2} \int_{x_{\|,} x_{\|}^{\prime}} \xi_{A}\left(x_{\|}\right) K_{A B}\left(x_{\|}, x_{\|}^{\prime}\right) \xi_{B}\left(x_{\|}^{\prime}\right)\right]
\end{aligned}
$$

$$
\begin{aligned}
& \frac{\mathcal{Z}^{C}[\psi]}{\mathcal{Z}_{0}^{C}} \\
& \quad=\int \mathcal{D} \xi_{I} \mathcal{D} \xi_{O} \exp \left[-\frac{1}{2} \int_{y_{\|}, y_{\|}^{\prime}} \xi_{A}\left(y_{\|}\right) T_{A B}\left(y_{\|}, y_{\|}^{\prime}\right) \xi_{B}\left(y_{\|}^{\prime}\right)\right] .
\end{aligned}
$$

These expressions are evidently different; indeed, even since the components of $x_{\|}$and $y_{\|}$have different dimensions. To make the comparison less awkward, we replace $\varphi$ by $x_{N}=r \varphi \in[-\pi r, \pi r)$. This implies that, at least in the $r \rightarrow \infty$ limit, the two partition functions should agree.
Performing that change of variables in (33), we obtain an additional $r^{2}$ factor, which leads to the conclusion that $\mathcal{Z}^{P}[\psi] / \mathcal{Z}_{0}^{P}=\mathcal{Z}^{C}[\psi] / \mathcal{Z}_{0}^{C}$ is equivalent to the equality between the kernels

$$
\frac{T_{A B}\left(y_{\|}, y_{\|}^{\prime}\right)}{r^{2}}=K_{A B}\left(x_{\|}, x_{\|}^{\prime}\right) .
$$

When $r$ is much larger than $d$ but still finite, the integral of one of the momenta in the calculation of $T_{A B}$ should be replaced by a sum over discrete momenta, as it happened for the $0 t$ th order, since one of the coordinates is periodic. We have at our disposal the calculation for one such system: two almost planar surfaces at a finite temperature $T$. In that kind of system, the fields are periodic in the imaginary time: $x_{0} \in[-1 / 2 T, 1 / 2 T]$, where $T$ is the temperature. Therefore, to use the results of such calculation, it is enough to replace $\beta$ by $2 \pi r$.

The second order of $\Gamma_{P} \equiv-\log \mathcal{Z}_{P}$, which may be extracted from [10], is:

$\Gamma_{P}^{(2)}[\psi]=\frac{1}{2 \beta} \sum_{n} \int_{\mathbf{k}_{\|} \equiv\left(k_{1}, k_{2}\right)} f^{(2)}\left(n, \mathbf{k}_{\|}\right)\left|\tilde{\eta}_{P}\left(n, \mathbf{k}_{\|}\right)\right|^{2}$,

with

$\tilde{\eta}_{P}\left(n, \mathbf{k}_{\|}\right)=\int_{\left(x_{0}, x_{1}, x_{2}\right) \equiv\left(x_{0}, \mathbf{x}_{\|}\right)} \eta_{P}\left(x_{0}, \mathbf{x}_{\|}\right) e^{-i \mathbf{k}_{\| \mid} \cdot \mathbf{x}_{\|}} e^{-i(n / r) x_{0}}$

and

$$
\begin{aligned}
f^{(2)}\left(n, \mathbf{k}_{\|}\right)=- & \frac{1}{\pi r d^{4}} \sum_{m} \int_{\mathbf{p}_{\|} \equiv\left(p_{1}, p_{2}\right)} \sqrt{(m d / r)^{2}+\mathbf{p}_{\|}^{2}} \\
& \times \sqrt{[(m+n) d / r]^{2}+\left(\mathbf{p}_{\|}+\mathbf{l}_{\|}\right)^{2}} \\
& \times \frac{1}{1-e^{-2 \sqrt{(m d / r)^{2}+\mathbf{p}_{\|}^{2}}}} \\
& \times \frac{1}{e^{2 \sqrt{[(m+n) d / r]^{2}+\left(\mathbf{p}_{\|}+\mathbf{I}_{\|}\right)^{2}}}-1},
\end{aligned}
$$

with $\mathbf{l}_{\|}=d \mathbf{k}_{\|}$.

Let us apply (III A 2) to a concrete example, that of two eccentric cylinders. This will allow us to find the explicit form of the function $Z_{2}$ involved in the proposed DE. We consider the external cylinder to be perturbed by a function $\eta_{C}(\varphi)=\epsilon \cos \varphi$. In the limit $\epsilon \ll d$, this describes two slightly eccentric cylinders, whose axes are separated by a distance $\epsilon$.

To obtain the interaction energy, we need $\tilde{\eta}_{P}\left(n, \mathbf{k}_{\|}\right)$. Setting $\eta_{C}(\varphi)=\eta_{P}\left(x_{N}\right)$, we see that

$\left|\tilde{\eta}_{P}\left(n, \mathbf{k}_{\|}\right)\right|^{2}=(2 \pi)^{2} r^{2} L^{2} \delta\left(\mathbf{k}_{\|}\right) \pi^{2} \epsilon^{2}\left(\delta_{n, 1}+\delta_{n,-1}\right)$. 
Thus:

$$
\lim _{L \rightarrow \infty} \frac{\Gamma_{P}^{(2)}[\psi]}{L^{2}}=\frac{\pi r}{4} \epsilon^{2}\left[f^{(2)}(1, \mathbf{0})+f^{(2)}(-1, \mathbf{0})\right] .
$$

Then we can use polar coordinates to perform the integral over $\mathbf{p}_{\|}$in Eq. (37). Defining $\rho=x /(\alpha-1)$, with $\alpha=b / a$, we may then write the second order in $\eta$ of the interaction energy per unit length, as

$$
\begin{aligned}
\mathcal{E}_{0}^{(2) l}[\psi]= & -\frac{\epsilon^{2}}{8 \pi a^{4}} \sum_{m} \int_{0}^{\infty} \mathrm{d} \rho \frac{\sqrt{[2 m /(\alpha+1)]^{2}+\rho^{2}}}{1-e^{-2(\alpha-1)} \sqrt{[2 m /(\alpha+1)]^{2}+\rho^{2}}} \\
& \times\left[\frac{\sqrt{[2(m+1) /(\alpha+1)]^{2}+\rho^{2}}}{e^{2(\alpha-1) \sqrt{[2(m+1) /(\alpha+1)]^{2}+\rho^{2}}}-1}\right. \\
& \left.+\frac{\sqrt{[2(m-1) /(\alpha+1)]^{2}+\rho^{2}}}{e^{2(\alpha-1) \sqrt{[2(m-1) /(\alpha+1)]^{2}+\rho^{2}}}-1}\right]
\end{aligned}
$$

which in the limit $\alpha \approx 1(d \ll r)$ reduces to:

$$
\begin{aligned}
\mathcal{E}_{0}^{(2) l}[\psi]= & -\frac{\epsilon^{2}}{8 \pi a^{4}} \sum_{m} \int_{0}^{\infty} \mathrm{d} \rho \frac{\sqrt{m^{2}+\rho^{2}}}{1-e^{-2(\alpha-1) \sqrt{m^{2}+\rho^{2}}}} \\
& \times\left[\frac{\sqrt{(m+1)^{2}+\rho^{2}}}{e^{2(\alpha-1) \sqrt{(m+1)^{2}+\rho^{2}}}-1}+\frac{\sqrt{(m-1)^{2}+\rho^{2}}}{e^{2(\alpha-1) \sqrt{(m-1)^{2}+\rho^{2}}}-1}\right] .
\end{aligned}
$$

The value of $\mathcal{E}^{(2) l}[\psi]$ thus obtained may be compared with the second order term which follows from the exact result in [15], where it is denoted as $E^{T M} / L$, since it corresponds to the transverse magnetic mode of the EM field. The second order of this exact solution is given by:

$$
\begin{aligned}
\frac{E^{T M(2)}}{L}= & -\frac{\epsilon^{2}}{4 \pi a^{4}} \sum_{n} \int_{0}^{\infty} \mathrm{d} \rho \rho^{3} \frac{1}{1-\mathcal{D}_{n, n}^{T M, c c}} \\
& \times\left[\mathcal{D}_{n}^{T M}+\frac{\mathcal{N}_{n}^{T M}}{1-\mathcal{D}_{n+1, n+1}^{T M, c c}}\right]
\end{aligned}
$$

where

$$
\begin{aligned}
\mathcal{D}_{n}^{T M} & =\frac{\mathcal{D}_{n, n}^{T M, c c}}{2}+\frac{I_{n}(\rho)}{4 K_{n}(\rho)}\left[\frac{K_{n-1}(\alpha \rho)}{I_{n-1}(\alpha \rho)}+\frac{K_{n+1}(\alpha \rho)}{I_{n+1}(\alpha \rho)}\right] \\
\mathcal{N}_{n}^{T M} & =\frac{I_{n}(\rho) I_{n+1}(\rho)}{4 K_{n}(\rho) K_{n+1}(\rho)}\left[\frac{K_{n 1}(\alpha \rho)}{I_{n 1}(\alpha \rho)}+\frac{K_{n+1}(\alpha \rho)}{I_{n+1}(\alpha \rho)}\right]^{2} \\
\mathcal{D}_{n, n}^{T M, c c} & =\frac{I_{n}(\rho) K_{n}(\alpha \rho)}{K_{n}(\rho) I_{n}(\alpha \rho)},
\end{aligned}
$$

and where $\epsilon$ is again the eccentricity of the cylinders. To perform the comparison with our approximate expression, we first divided them by $\epsilon^{2} / a^{4}$.
TABLE I. Comparison, for different values of $\alpha$, between (41) and (42). The fourth column contains the error, defined as: $100 \times 2 \times\left(E^{T M(2)} / L-\mathcal{E}_{0}^{(2) l}\right) /\left(E^{T M(2)} / L+\mathcal{E}_{0}^{(2) l}\right)$.

\begin{tabular}{lccl}
\hline \hline$\alpha$ & $\left|\mathcal{E}_{0}^{(2) l}\right| /\left(\epsilon^{2} / a^{4}\right)$ & $\left|E^{T M(2)} / L\right| /\left(\epsilon^{2} / a^{4}\right)$ & Error $(\%)$ \\
\hline 1.1 & 12933.6 & 13557.6 & 4.7 \\
1.01 & $6.60 \times 10^{8}$ & $6.62 \times 10^{8}$ & 0.3 \\
1.001 & $7.2034 \times 10^{12}$ & $7.2058 \times 10^{12}$ & 0.03 \\
1.0001 & $7.21003 \times 10^{16}$ & $7.21012 \times 10^{16}$ & 0.001 \\
1.00001 & $7.21010 \times 10^{20}$ & $7.21012 \times 10^{20}$ & 0.0003 \\
\hline \hline
\end{tabular}

Performing the sums and integrals numerically, we have found that while (40) is indeed a better approximation than (41) for $\alpha=1.1$ and bigger, they are quite similar for smaller values. In Table I, we show the comparison between (41) and (42). The error in the approximate expression decreases when $\alpha \rightarrow 1$, staying below $0.3 \%$ when $\alpha<1.01$.

Finally, let us obtain the function $Z_{2}$ of the DE for this case, based also on the example of eccentric cylinders. For $\psi=\psi(\varphi)$, this expansion reduces to:

$E_{0}[\psi]=\int_{0}^{2 \pi} \mathrm{d} \varphi\left[V(\psi(\varphi))+Z_{2}(\psi(\varphi))\left(\frac{\partial \psi}{\partial \varphi}\right)^{2}\right]$

Now, setting $\psi(\varphi)=b+\eta(\varphi)$, with $|\eta| \ll b-a$, and expanding up to second order in $\eta$ :

$$
\begin{aligned}
E_{0}[b+\eta] \simeq & \int_{0}^{2 \pi} \mathrm{d} \varphi\left[V(b)+V^{\prime}(b) \eta(\varphi)+\frac{1}{2} V^{\prime \prime}(b) \eta^{2}(\varphi)\right. \\
& \left.+Z_{2}(b)\left(\frac{\partial \eta}{\partial \varphi}\right)^{2}\right] .
\end{aligned}
$$

Setting now $\eta(\varphi)=\epsilon \cos \varphi$, we arrive to:

$$
E_{0}[\psi] \simeq 2 \pi V(b)+\frac{\pi \epsilon^{2}}{2} V^{\prime \prime}(b)+\pi \epsilon^{2} Z_{2}(b) .
$$

Hence we can extract the function $Z_{2}(b)$ :

$$
Z_{2}(b)=\frac{1}{\pi \epsilon^{2}}\left(E_{0}[\psi]-2 \pi V(b)-\frac{\pi \epsilon^{2}}{2} V^{\prime \prime}(b)\right) .
$$

At this point, it is useful to separate the total energy $E_{0}$ as a sum of its different orders in $\epsilon$. Doing so, we can see that the zeroth-order term equals $2 \pi V(b)$. Hence, we are left with the following reduced expression:

$$
Z_{2}(b)=\frac{1}{\pi \epsilon^{2}}\left(E_{0}^{(2)}[\psi]-\frac{\pi \epsilon^{2}}{2} V^{\prime \prime}(b)\right)
$$

where $E_{0}^{(2)}[\psi]$ is the second order term (in $\epsilon$ ) of the energy. 
At this time, we note that we can extract this term either from [15], or from the approximation (41). We just need to evaluate the second derivative of $V(b)$ with respect to $b$. Using our approximate expression for concentric cylinders we obtain:

$$
V(b)=\frac{L}{4 \pi} \sum_{n} \int_{\mathbf{k}_{\|}} \log \left(1-e^{-2 d \sqrt{(n / r)^{2}+\mathbf{k}_{\|}^{2}}}\right) .
$$

Finally, we arrive to:

$$
\begin{aligned}
V^{\prime \prime}(b)= & -\frac{L}{8 \pi^{2} r^{4}} \sum_{n} \int_{0}^{\infty} \mathrm{d} \rho \rho\left(\rho^{2}+n^{2}\right) \\
& \times \operatorname{cosech}^{2}\left((\alpha-1) \sqrt{\rho^{2}+n^{2}}\right),
\end{aligned}
$$

with $\alpha=b / a$.

$Z_{2}(b)$ may then be obtained by using our results for the energy.

$$
\begin{aligned}
Z_{2}(b)= & \frac{L}{4 \pi^{2}} \sum_{n} \int_{0}^{\infty} \mathrm{d} \rho\left\{\frac{\rho\left(\rho^{2}+n^{2}\right)}{4 r^{4}} \operatorname{cosech}^{2}\left((\alpha-1) \sqrt{\rho^{2}+n^{2}}\right)\right. \\
& \left.-\frac{1}{2 a^{4}} \frac{\sqrt{(m+1)^{2}+\rho^{2}}}{1-e^{-2(\alpha-1) \sqrt{m^{2}+\rho^{2}}}}\left[\frac{\sqrt{(m+1)^{2}+\rho^{2}}}{e^{2(\alpha-1) \sqrt{(m+1)^{2}+\rho^{2}}}-1}+\frac{\sqrt{(m-1)^{2}+\rho^{2}}}{e^{2(\alpha-1) \sqrt{(m-1)^{2}+\rho^{2}}}-1}\right]\right\} .
\end{aligned}
$$

To sum up, equations (46), (51) and (53) determine the second order DE for the Dirichlet case.

\section{B. Scalar field: Neumann conditions}

The same calculations can be performed when Neumann conditions are imposed. For this purpose, we choose the following boundary conditions:

$$
\begin{gathered}
\left.\partial_{\rho} \phi(y)\right|_{s_{I}}=0 \\
\left.\partial_{n} \phi(y)\right|_{s_{O}}=0,
\end{gathered}
$$

with $\partial_{n} \equiv n^{\mu} \partial_{\mu}$ and $\partial_{\mu} \equiv \frac{\partial}{\partial x^{\mu}}$, where $n^{\mu}\left(y_{\|}\right)$is a unit vector perpendicular to $s_{O}$, and $x^{\mu}$ are usual Cartesian coordinates:

$$
n^{\mu}\left(y_{\|}\right)=\frac{N^{\mu}\left(y_{\|}\right)}{\left|N^{\mu}\left(y_{\|}\right)\right|},
$$

with

$$
N^{\mu}\left(y_{\|}\right)=\left\{\begin{array}{ll}
0 & (\mu=0) \\
\partial_{\varphi} \psi \sin \varphi+\psi \cos \varphi & (\mu=1) \\
-\partial_{\varphi} \psi \cos \varphi+\psi \sin \varphi & (\mu=2) \\
-\psi \partial_{z} \psi & (\mu=3)
\end{array} .\right.
$$

Again, we can include the boundary condition using functionals $\delta_{I}[\phi]$ and $\delta_{O}[\phi]$ :

$$
\begin{aligned}
\delta_{I}[\phi] & =\int \mathcal{D} \xi_{I} \exp \left[i \int_{y} \xi_{I}(y) \delta(\rho-a) \partial_{\rho} \phi(y)\right] \\
\delta_{O}[\phi] & =\int \mathcal{D} \xi_{O} \exp \left[i \int_{y} \sqrt{g\left(y_{\|}\right)} \xi_{O}(y) \frac{\delta\left(\rho-\psi\left(y_{\|}\right)\right)}{\rho} \partial_{n} \phi(y)\right] .
\end{aligned}
$$

Following analogous steps to those in Sec. III A, $\mathcal{Z}$ may be written in a familiar form:

$$
\frac{\mathcal{Z}[\psi]}{\mathcal{Z}_{0}}=\int \mathcal{D} \xi_{I} \mathcal{D} \xi_{O} \exp \left[-\frac{1}{2} \int_{y_{\|}, y_{\|}^{\prime}} \xi_{A}\left(y_{\|}\right) N_{A B}\left(y_{\|}, y_{\|}^{\prime}\right) \xi_{B}\left(y_{\|}^{\prime}\right)\right]
$$

where

$N_{I I}\left(y_{\|}, y_{\|}^{\prime}\right)=a^{2}\left[\partial_{\rho} \partial_{\rho}^{\prime}\left\langle y\left|\left(-\partial^{2}\right)^{-1}\right| y^{\prime}\right\rangle\right]_{\rho=\rho^{\prime}=a}$

$N_{I O}\left(y_{\|}, y_{\|}^{\prime}\right)=a\left[\partial_{\rho} \partial_{N}^{\prime}\left\langle y\left|\left(-\partial^{2}\right)^{-1}\right| y^{\prime}\right\rangle\right]_{\rho=a, \rho^{\prime}=\psi\left(y_{\|}^{\prime}\right)}$

$N_{O I}\left(y_{\|}, y_{\|}^{\prime}\right)=a\left[\partial_{N} \partial_{\rho}^{\prime}\left\langle y\left|\left(-\partial^{2}\right)^{-1}\right| y^{\prime}\right\rangle\right]_{\rho=\psi\left(y_{\|}\right), \rho^{\prime}=a}$

$N_{O o}\left(y_{\|}, y_{\|}^{\prime}\right)=\left[\partial_{N} \partial_{N}^{\prime}\left\langle y\left|\left(-\partial^{2}\right)^{-1}\right| y^{\prime}\right\rangle\right]_{\rho=\psi\left(y_{\|}\right), \rho^{\prime}=\psi\left(y_{\|}^{\prime}\right)}$,

with $\partial_{\rho}^{\prime} \equiv \frac{\partial}{\partial \rho^{\prime}}$ and $\partial_{N}^{\prime} \equiv N^{\mu}\left(y_{\|}^{\prime}\right) \partial_{\mu}$. As before, this allows us to calculate the first orders in $\eta$ of the interaction energy.

\section{Order 0 in $\eta$}

We start again with the order 0 in $\eta$. Following similar steps as before, we obtain that the matrix $\mathbb{N}\left(y_{\|}, y_{\|}^{\prime}\right)$ may be written as:

$\mathbb{N}\left(y_{\|}, y_{\|}^{\prime}\right)=\int_{\mathbf{k}_{\|}} e^{i \mathbf{k}_{\|} \cdot\left(\mathbf{y}_{\|}-\mathbf{y}_{\|}\right)} \frac{1}{2 \pi} \sum_{n} e^{i n\left(\varphi-\varphi^{\prime}\right)} \tilde{\mathbb{N}}\left(n, \mathbf{k}_{\|}\right)$,

where 


$$
\begin{aligned}
& \tilde{\mathbb{N}}\left(n, \mathbf{k}_{\|}\right) \\
& \quad=\mathbf{k}_{\|}^{2}\left(\begin{array}{cc}
a^{2} I_{n}^{\prime}\left(\left|\mathbf{k}_{\|}\right| a\right) K_{n}^{\prime}\left(\left|\mathbf{k}_{\|}\right| a\right) & a b I_{n}^{\prime}\left(\left|\mathbf{k}_{\|}\right| a\right) K_{n}^{\prime}\left(\left|\mathbf{k}_{\|}\right| b\right) \\
a b I_{n}^{\prime}\left(\left|\mathbf{k}_{\|}\right| a\right) K_{n}^{\prime}\left(\left|\mathbf{k}_{\|}\right| b\right) & b^{2} I_{n}^{\prime}\left(\left|\mathbf{k}_{\|}\right| b\right) K_{n}^{\prime}\left(\left|\mathbf{k}_{\|}\right| b\right)
\end{array}\right) .
\end{aligned}
$$

This matrix leads to the interaction energy per unit area:

$$
\begin{aligned}
\mathcal{E}_{0}^{(0)} & =\frac{1}{4 \pi r} \int_{\mathbf{k}_{\|}} \sum_{n} \log \operatorname{det} \tilde{\mathbb{N}}\left(n,\left|\mathbf{k}_{\|}\right|\right) \\
& =\frac{1}{4 \pi r} \int_{\mathbf{k}_{\|}} \sum_{n} \log \left[1-\frac{I_{n}^{\prime}\left(\left|\mathbf{k}_{\|}\right| a\right) K_{n}^{\prime}\left(\left|\mathbf{k}_{\|}\right| b\right)}{I_{n}^{\prime}\left(\left|\mathbf{k}_{\|}\right| b\right) K_{n}^{\prime}\left(\left|\mathbf{k}_{\|}\right| a\right)}\right],
\end{aligned}
$$

which coincides with the exact solution for concentric cylinders, computed in [15].

On the other hand, one again expects the matrices $\mathbb{U}$ and $\mathbb{N}$ to satisfy an analogous relation to (34) in the limit $d \ll r$, i.e.,

$$
\frac{\mathbb{N}\left(y_{\|}, y_{\|}^{\prime}\right)}{r^{2}} \approx \mathbb{U}\left(x_{\|}, x_{\|}^{\prime}\right),
$$

where $\mathbb{U}$ is the equivalent to the matrix $\mathbb{K}$ in (32), in the case where Neumann conditions are imposed. At order 0 in $\eta$, this relation can be proved approximating the Bessel functions as in (26), which gives:

$$
\mathbb{N}^{(0)}\left(y_{\|}, y_{\|}^{\prime}\right) \approx-\frac{r}{4 \pi} \int_{\mathbf{k}_{\|}} \sum_{n} e^{i k_{\|}\left(y_{\|}-y_{\|}^{\prime}\right)}\left|k_{\|}\right|\left(\begin{array}{cc}
1 & e^{-d\left|k_{\|}\right|} \\
e^{-d\left|k_{\|}\right|} & 1
\end{array}\right),
$$

where, as before, $k_{\|} \equiv\left(\omega_{n}, \mathbf{k}_{\|}\right)$, with $\omega_{n}=n / r$. Finally, we can use this to calculate the interaction energy per unit length:

$$
\mathcal{E}_{0}^{l} \approx \frac{1}{2} \int_{\mathbf{k}_{\|}} \sum_{n} \log \left(1-e^{-2 d \sqrt{(n / r)^{2}+\mathbf{k}_{\|}^{2}}}\right) .
$$

As it happens when Dirichlet conditions are imposed, if we divide this expression by $2 \pi r$ and take the limit $r \rightarrow \infty$, we again obtain the energy density per unit area between parallel planes. On the other hand, Eq. (69) leads to the same value of $\mathcal{E}_{0}^{l}$ obtained with Dirichlet conditions, in the limit $d \ll r$. Then, in that limit, the energy per unit length of the electromagnetic field coupled to perfect conductors shaped as $s_{I}$ and $s_{O}$ must be the following:

$$
\begin{aligned}
\mathcal{E}_{0}^{l(E M)} & =\mathcal{E}_{0}^{l(\text { Dirichlet })}+\mathcal{E}_{0}^{l(\text { Neumann })}=2 \mathcal{E}_{0}^{l(\text { Dirichlet })} \\
& \approx \int_{\mathbf{k}_{\|}} \sum_{n} \log \left(1-e^{-2 d \sqrt{(n / r)^{2}+\mathbf{k}_{\|}^{2}}}\right),
\end{aligned}
$$

which coincides with the limit $d \ll r$ of the exact solution (see [15]).

\section{Order 2 in $\eta$}

We consider here the second order term. We shall see that, when Neumann boundary conditions are imposed on $s_{I}$ and $s_{O}$, depending on the variables upon which $\eta$ depends, the energy can have nonanalytic properties that may render the DE not applicable in certain cases.

The second order term from $\Gamma_{P}[\psi]$, calculated in [10], is

$$
\Gamma_{P}^{(2)}[\psi]=\frac{1}{2 \beta} \sum_{n} \int_{\mathbf{k}_{\|}} g^{(2)}\left(n, \mathbf{k}_{\|}\right)\left|\tilde{\eta}_{P}\left(n, \mathbf{k}_{\|}\right)\right|^{2},
$$

with

$$
\begin{aligned}
g^{(2)}\left(n, \mathbf{k}_{\|}\right)= & -\frac{1}{\pi r d^{4}} \sum_{m} \int_{\mathbf{p}_{\|}} \frac{\left[m(m+n)(d / r)^{2}+\mathbf{p}_{\| \cdot}\left(\mathbf{p}_{\|}+\mathbf{l}_{\|}\right)\right]^{2}}{\sqrt{(m d / r)^{2}+\mathbf{p}_{\|}^{2}} \sqrt{[(m+n) d / r]^{2}+\left(\mathbf{p}_{\|}+\mathbf{l}_{\|}\right)^{2}}} \\
& \times \frac{1}{1-e^{-2 \sqrt{(m d / r)^{2}+\mathbf{p}_{\|}^{2}}}} \frac{1}{e^{2 \sqrt{[(m+n) d / r]^{2}+\left(\mathbf{p}_{\|}+\mathbf{l}_{\|}\right)^{2}}}-1}
\end{aligned}
$$

where $\mathbf{I}_{\|}=d \mathbf{k}_{\|}$, and $\beta=2 \pi r$ as before.

The expansion of $g^{(2)}$ close to zero momentum can be used to obtain the different orders in derivatives of $\eta$, which is not possible if $g^{(2)}\left(n, \mathbf{k}_{\|}\right)$is not analytic in a neighbourhood of $\left(n, \mathbf{k}_{\|}\right)=(0, \mathbf{0})$. One way to verify this kind of issue is to study the behavior of the function $g^{(2)}\left(0, \mathbf{k}_{\|}\right)$ around $\mathbf{k}_{\|}=0$. Examining Eq. (72), we can see that the terms with $m \neq 0$ will be analytic, since they are integrals of quotients of analytic integrable functions that do not vanish. We still have to see the term with $m=0$, for which we define $g\left(\mathbf{k}_{\|}\right)$as the term with $m=0$ in (72) when $n=0$. Namely,

$$
g\left(\mathbf{k}_{\|}\right)=-\frac{1}{\pi r d^{4}} \int_{\mathbf{p}_{\|}} \frac{\left[\mathbf{p}_{\|} \cdot\left(\mathbf{p}_{\|}+\mathbf{l}_{\|}\right)\right]^{2}}{\left|\mathbf{p}_{\|} \| \mathbf{p}_{\|}+\mathbf{l}_{\|}\right|} \frac{1}{1-e^{-2\left|\mathbf{p}_{\|}\right|}} \frac{1}{e^{2\left|\mathbf{p}_{\|}+\mathbf{I}_{\|}\right|}-1}
$$

A long calculation that involves dimensional regularization proves that, close to $\mathbf{k}_{\|}=\mathbf{0}$, this function behaves as 


$$
g\left(\mathbf{k}_{\|}\right) \approx g(\mathbf{0})-\frac{1}{32 \pi^{2} r} \frac{\mathbf{k}_{\|}^{2}}{d^{2}} \log \left(\mathbf{k}_{\|}^{2} d^{2}\right)+\mathcal{O}\left(\mathbf{k}_{\|}^{2}\right),
$$

where the term of order $\mathbf{k}_{\|}^{2}$ is finite.

Replacing it in Eq. (71), a term proportional to $\mathbf{k}_{\| \mid}^{2} \log \left(\mathbf{k}_{\|}^{2} d^{2}\right)$ would give rise to contributions to the energy proportional to

$$
\int_{\mathbf{x}_{\|}} \int_{\varphi, z} \eta(\varphi, z) \partial_{z}^{2} \log \left(-d^{2} \partial_{z}^{2}\right) \eta(\varphi, z)
$$

and therefore the proposed DE would not be applicable to this case. If $\eta$ does not depend on $z$, however, $\left|\tilde{\eta}_{P}\left(n, \mathbf{k}_{\|}\right)\right|^{2}$ results to be proportional to $\delta\left(\mathbf{k}_{\|}\right)$, which nullifies the contribution of terms such as $\mathbf{k}_{\|}^{2} \log \left(\mathbf{k}_{\|}^{2} d^{2}\right)$ whose limit as $\mathbf{k}_{\|} \rightarrow \mathbf{0}$ is 0 . Consequently, the applicability of the DE depends in this case on the analyticity of $g^{(2)}(n, \mathbf{0})$ as a function of $n$. On the other hand, these problems with $\mathbf{k}_{\|}$do not appear when Dirichlet conditions are fixed, since in that case the zero-momentum expansion of the function equivalent to $g\left(\mathbf{k}_{\|}\right)$has only the $\mathcal{O}\left(\mathbf{k}_{\|}^{2}\right)$ term, apart from the constant one.

\section{CONCLUSIONS}

We have constructed a version of the DE which is suitable for application to cylindrical surfaces, and for a rather general interaction. That expansion has then been applied to the Casimir effect at zero temperature, for a real scalar field satisfying either Dirichlet or Neumann conditions on two surfaces. We have shown how, in the limit where the DE yields approximate results, one can determine the functions appearing in the DE approximation just from the knowledge of results for planar surfaces at finite temperature. The role of the temperature is here of course rather fictitious, since it is used (via the Matsubara formalism) to have a periodic coordinate. We have checked numerically the intuitive idea that, when two cylindrical surfaces are very close in comparison with the curvature radius, the predictions coming from exact results are essentially the same as the ones coming from planes with a periodic coordinate. We may say that, at least to the second order, the DE is sensitive to the topology (periodicity) of the system, albeit not to the detailed geometry (the metric tensor).

In the Neumann case, the same known nonanalyticity found at finite temperature for planes arises. However, one can also show explicitly that, if the surfaces are translation invariant in $z$, the nonanalyticity disappears from the final expression.

\section{ACKNOWLEDGMENTS}

We are thankful to F. D. Mazzitelli for useful discussions that led to the improvement of this work. We acknowledge financial support from ANPCyT, CONICET, and UNCuyo (Argentina). We are especially grateful to Instituto Balseiro, where most of this work has been done.

\section{APPENDIX: DERIVATION OF THE DE FOR TWO CYLINDRICAL SURFACES}

We derive here the DE shown in Sec. II B for cylindrical surfaces [Eqs. (6) to (8)]. For this, we consider two surfaces: $I$, which corresponds to $\rho=a$, and $O$ to $\psi(\varphi, z)=$ $b+\eta(\varphi, z)$, with $b>a$.

As in the case of Cartesian coordinates, we begin by assuming that the functional $F[\psi]$, that represents the interaction energy, may be expanded in a functional Taylor series:

$F[\psi]=\sum_{n \geq 0} \frac{1}{n !} \int_{x_{1}, \ldots, x_{n}} \Gamma^{(n)}\left(x_{1}, \ldots, x_{n}\right) \eta\left(x_{1}\right) \ldots \eta\left(x_{n}\right)$,

where we have used a shorthand notation $x \equiv(\varphi, z)$ for the integration variables. Namely,

$\int_{x_{1}, \ldots, x_{n}} \ldots \equiv \int_{-\infty}^{\infty} \mathrm{d} z_{1} \int_{-\pi}^{\pi} \mathrm{d} \varphi_{1} \ldots \int_{-\infty}^{\infty} \mathrm{d} z_{n} \int_{-\pi}^{\pi} \mathrm{d} \varphi_{n} \ldots$

Since we want to deal with smooth functions, $\eta$ (and therefore $\psi$ ) must be $2 \pi$-periodic in its angular argument $\varphi$.

The functional derivatives evaluated at the expansion point have been denoted by $\Gamma^{(n)}\left(x_{1}, \ldots, x_{n}\right)=$ $\left.\left[\frac{\delta^{n} F}{\delta \eta\left(x_{1}\right) \ldots \delta \eta\left(x_{n}\right)}\right]\right]_{\eta \equiv 0}, \forall n \geq 1$, and $\Gamma^{(0)} \equiv F[b]$. Since those functional derivatives are evaluated at $\psi=b$, they must exhibit the same symmetries that leave the geometry of that system (two concentric circular cylinders) invariant. The symmetry group contains translations in $z$ and rotations in $\varphi$. Therefore, we conclude that $\Gamma^{(1)}$ can only be a constant function, and that $\Gamma^{(2)}\left(x_{1}, x_{2}\right)$ may only depend on the difference $x_{1}-x_{2}$. Furthermore, for $n>2$, one can show that $\Gamma^{(n)}$ may be written in terms of just $n-1$ independent variables, for instance, $\left(x_{1}-x_{2}, x_{2}-x_{3}, \ldots, x_{n-2}-x_{n-1}\right.$, $\left.x_{1}+\cdots+x_{n-1}-(n-1) x_{n}\right)$.

To proceed, as in the case of Cartesian coordinates, we assume that the radius $b$ has been chosen in such a way that $\int_{x} \eta(x)=0$; with this choice, the $n=1$ term vanishes. Thus, introducing the Fourier transform of $\eta$ :

$$
\eta(\varphi, z)=\frac{1}{2 \pi} \sum_{n=-\infty}^{\infty} \int_{k} \tilde{\eta}(k, n) e^{i k z} e^{i n \varphi}
$$

with $\int_{k} \equiv \int_{-\infty}^{\infty} \frac{d k}{2 \pi}$, we see that: 


$$
\begin{aligned}
F[\psi]= & F[b] \\
& +\sum_{n \geq 2} \frac{1}{(2 \pi)^{n}} \sum_{m_{1}, \ldots, m_{n}} \int_{k_{1}, \ldots, k_{n}} h^{(n)}\left(k_{1}, \ldots, k_{n}, m_{1}, \ldots, m_{n}\right) \\
& \times \tilde{\eta}\left(k_{1}, m_{1}\right) \ldots \tilde{\eta}\left(k_{n}, m_{n}\right) \delta\left(k_{1}+\cdots+k_{n}\right) \\
& \times \delta\left(m_{1}+\cdots+m_{n}\right),
\end{aligned}
$$

where $h^{(n)}\left(k_{1}, \ldots, k_{n}, m_{1}, \ldots, m_{n}\right)$ are the symmetrized form factors.

Based on the previous expressions, we now deal with the zeroth and second order terms in the DE (the first order one vanishes by the proper choice of $b$ ).

\section{Zeroth order in derivatives}

When $\eta$ becomes sufficiently smooth, $\tilde{\eta}(k, m)$ is concentrated around zero momentum, namely, $(k=0, m=0)$. The leading term in this expansion amounts to keeping just that component, namely, to replacing in (A4) the form factors by their zero-momentum limits.

Hence,

$$
\begin{aligned}
F[\psi] \simeq & F_{0}[\psi] \\
\equiv & F[b]+\sum_{n \geq 2} h^{(n)}(0, \ldots, 0) \int_{x_{1}, \ldots, x_{n}} \frac{1}{(2 \pi)^{n}} \\
& \times \sum_{m_{1}, \ldots, m_{n}} \int_{k_{1}, \ldots, k_{n}} \eta\left(x_{1}\right) \ldots \eta\left(x_{n}\right) \\
& \times e^{-i \sum_{j=1}^{n} k_{j} z_{j}} e^{-i \sum_{j=1}^{n} m_{j} \varphi_{j}} \delta\left(k_{1}+\cdots+k_{n}\right) \\
& \times \delta\left(m_{1}+\cdots+m_{n}\right) .
\end{aligned}
$$

By taking into account the presence of the delta functions, we can perform both the integral over $k_{n}$ and the sum over $m_{n}$, obtaining:

$$
F_{0}[\psi]=F[b]+\int_{x} \sum_{n \geq 2} \frac{h^{(n)}(0, \ldots, 0)}{(2 \pi)^{2}} \eta(x)^{n}
$$

Let us now deal with the evaluation of the sum

$$
\sum_{n \geq 2} h^{(n)}(0, \ldots, 0)(\eta(x))^{n} /(2 \pi)^{2}
$$

as a function of $x$, considering a constant $\eta \equiv \eta_{0}$, for which we get

$$
\mathcal{F}_{0}\left(b+\eta_{0}\right)=\mathcal{F}_{0}(b)+\frac{1}{b} \sum_{n \geq 2} \frac{h^{(n)}(0, \ldots, 0)}{(2 \pi)^{2}} \eta_{0}^{n},
$$

where $\mathcal{F}_{0}(b)$ denotes the function:

$$
\mathcal{F}_{0}(b)=\lim _{L \rightarrow \infty} \frac{F[b]}{S_{b, L}},
$$

where $S_{b, L}$ denotes the total area of the cylinder $r=b$ and length $L$.

Hence, we extract the relation:

$\frac{1}{b} \sum_{n \geq 2} \frac{h^{(n)}(0, \ldots, 0)}{(2 \pi)^{2}} \eta(x)^{n}=\mathcal{F}_{0}(b+\eta(x))-\mathcal{F}_{0}(b)$.

Using the expression above in (A6), we see that:

$$
\begin{aligned}
F_{0}[\psi] & =\int_{x} b \mathcal{F}_{0}(b+\eta(x)) \\
& =\int_{-\pi}^{\pi} \mathrm{d} \varphi \int_{-\infty}^{\infty} \mathrm{d} z b \mathcal{F}_{0}(b+\eta(\varphi, z)) .
\end{aligned}
$$

Note that the expression above is quite different to the would-be zeroth order result for the DE based on planar surfaces. In fact, that would mean to integrate the energy per unit area for planes, $\mathcal{F}_{0}^{P}$, over a planar surface $L$. Indeed, for a physical problem described by two surfaces defined in cylindrical coordinates by $\rho=r_{1}$ and $\rho=$ $r_{2}+\eta(z, \varphi)$, this planar PFA yields

$$
F_{0}^{P}=\int_{S} \mathcal{F}_{0}^{P}\left(r_{2}+\eta(z, \varphi)-r_{1}\right),
$$

where $S$ is some intermediate surface, and, clearly, the result will in general depend on the choice of the surface $S$. This is not so for $F_{0}$.

The reason for the difference between the two approaches is of course the fact that the density $\mathcal{F}_{0}$ generally depends on both $b$ and $a$ independently, not just on their difference like it necessarily happens for $\mathcal{F}_{0}^{P}$. As a simple example of this situation, we recall the case of the electrostatic interaction between two conducting surfaces, held at a constant potential difference, where $\mathcal{F}_{0}$ is a function of $\log (b / a)$.

\section{Higher orders}

To obtain higher order terms in the expansion in derivatives, we need the corresponding terms in the Taylor expansion of the momentum space form factors at zero momentum. Assuming the expansion is well defined,

$$
\begin{aligned}
h^{(n)} & \left(k_{1}, \ldots, k_{n}, m_{1}, \ldots, m_{n}\right) \\
= & h^{(n)}(0, \ldots, 0)+A^{(n) i} k_{i}+B^{(n) i} m_{i}+C^{(n) i j} k_{i} k_{j} \\
& +D^{(n) i j} m_{i} m_{j}+E^{(n) i j} m_{i} k_{j}+\cdots .
\end{aligned}
$$

Besides, the variables $m_{i}$ are integers. However, the analyticity of $h^{(n)}$ for $m_{i}$ regarded as real variables is a 
sufficient condition for the validity and unicity of the expansion (A13).

To study the consequences of (A13), we may first note that the coefficients $C^{(n) i j}$ and $D^{(n) i j}$ can be regarded as invariant under the exchange of arbitrary $i$ and $j$, since they are multiplied respectively by $k_{i} k_{j}$ and $m_{i} m_{j}$. On the other hand, $h^{(n)}\left(\left\{k_{i}\right\},\left\{m_{i}\right\}\right)$ must be invariant under the exchange of any two pairs $\left(k_{l}, m_{l}\right)$ and $\left(k_{s}, m_{s}\right)$. Therefore, using (A13), we can calculate the difference between $h^{(n)}$ and the same factor when two such pairs are exchanged, up to order 2 in $\left\{m_{i}\right\},\left\{k_{i}\right\}$. This gives the following relation for every $l, s$ :

$$
\begin{aligned}
0= & \left(A^{l}-A^{s}\right)\left(k_{l}-k_{s}\right)+\left(B^{l}-B^{s}\right)\left(m_{l}-m_{s}\right)+\left(C^{l l}-C^{s s}\right)\left(k_{l} k_{l}-k_{s} k_{s}\right)+2 \sum_{i \neq s, l} k_{i}\left(k_{l}-k_{s}\right)\left(C^{i l}-C^{i s}\right) \\
& +2 \sum_{i \neq s, l} m_{i}\left(m_{l}-m_{s}\right)\left(D^{i l}-D^{i s}\right)+\sum_{j \neq s, l} k_{j}\left(m_{l}-m_{s}\right)\left(E^{l j}-E^{s j}\right) \\
& +\sum_{i \neq s, l}\left[m_{i}\left(k_{l}-k_{s}\right)\left(E^{i l}-E^{i s}\right)+\left(E^{l s}-E^{s l}\right)\left(m_{l} k_{s}-m_{s} k_{l}\right)+\left(E^{l l}-E^{s s}\right)\left(m_{l} k_{l}-m_{s} k_{s}\right)\right],
\end{aligned}
$$

where the indices $(n)$ have been omitted. Using this equation, we can obtain useful relations involving the coefficients $A^{i}, B^{i}, C^{i j}, D^{i j}$, and $E^{i j}$. For instance, setting $k_{i}=0$ except for $k_{l}$ and $k_{s}$, and every $m_{i}$ equal to 0 , we obtain:

$0=\left(A^{l}-A^{s}\right)\left(k_{l}-k_{s}\right)+\left(C^{l l}-C^{s s}\right)\left(k_{l} k_{l}-k_{s} k_{s}\right)$,

from where $A^{l}=A^{s}$ and $C^{l l}=C^{s s}$ for every $l, s$, since otherwise the functions $\left(k_{l}-k_{s}\right)$ and $\left(k_{l} k_{l}-k_{s} k_{s}\right)$ would be linearly dependent. In a similar fashion, another set of relations may be obtained:

$A^{l}=A^{s}, \quad B^{l}=B^{s}, \quad C^{l l}=C^{s s}, \quad D^{l l}=D^{s s}$,

$E^{l l}=E^{s s}, \quad E^{l s}=E^{s l} \quad \forall l, s$

$C^{r l}=C^{r s}, \quad D^{r l}=D^{r s}$,

$E^{r l}=E^{r s} \quad \forall l, s, r / l \neq s, s \neq r, l \neq r$.

Using this result, Eq. (A13) may be rendered as

$$
\begin{aligned}
h^{(n)} & \left(k_{1}, \ldots, k_{n}, m_{1}, \ldots, m_{n}\right) \\
= & h^{(n)}(0, \ldots, 0)+A^{(n)} \sum_{i} k_{i}+B^{(n)} \sum_{i} m_{i} \\
& +\left[C_{1}^{(n)} \sum_{i} k_{i} k_{i}+C_{2}^{(n)} \sum_{i>j} k_{i} k_{j}+D_{1}^{(n)} \sum_{i} m_{i} m_{i}\right. \\
& \left.+D_{2}^{(n)} \sum_{i>j} m_{i} m_{j}+E_{1}^{(n)} \sum_{i} m_{i} k_{i}+E_{2}^{(n)} \sum_{i>j} \frac{m_{i} k_{j}+m_{j} k_{i}}{2}\right] \\
& +\cdots
\end{aligned}
$$

Replacing this in Eq. (A4), we can see that the term $h^{(n)}(0, \ldots, 0)$ gives rise to the functional $F_{0}$ already calculated in (4). On the other hand, the linear terms are multiplied by $\delta\left(k_{1}+\cdots+k_{n}\right) \delta\left(m_{1}+\ldots m_{n}\right)$, and therefore their contribution vanishes. We are finally left with the order-2 terms, highlighted between brackets in (A18). Performing analogous steps to those followed in the previous section, we obtain that these produce a contribution $F_{2}[\psi]$ given by

$$
\begin{aligned}
F_{2}[\psi]= & -\frac{1}{(2 \pi)^{2}} \sum_{n \geq 2} \int_{x}\left[C_{1}^{(n)} n \eta(x)^{n-1} \partial_{z}^{2} \eta(x)+C_{2}^{(n)} n(n-1) \eta(x)^{n-2}\left(\partial_{z} \eta(x)\right)^{2}+D_{1}^{(n)} n \eta(x)^{n-1} \partial_{\varphi}^{2} \eta(x)\right. \\
& \left.+D_{2}^{(n)} n(n-1) \eta(x)^{n-2}\left(\partial_{\varphi} \eta(x)\right)^{2}+E_{1}^{(n)} n \eta(x)^{n-1} \partial_{z} \partial_{\varphi} \eta(x)+E_{2}^{(n)} n(n-1) \eta(x)^{n-2}\left(\partial_{z} \eta(x)\right)\left(\partial_{\varphi} \eta(x)\right)\right] .
\end{aligned}
$$

Now we can perform an integration by parts of the terms that are proportional to $C_{1}^{(n)}, D_{1}^{(n)}$ and $E_{1}^{(n)}$. For instance, for those proportional to $C_{1}^{(n)}$, we may do what follows:

$$
\begin{aligned}
\int_{-\pi}^{\pi} \mathrm{d} \varphi \int_{-\infty}^{\infty} \mathrm{d} z \eta(x)^{n-1} \partial_{z}^{2} \eta(x) & =-(n-1) \int_{-\pi}^{\pi} \mathrm{d} \varphi \int_{-\infty}^{\infty} \mathrm{d} z \eta(x)^{n-2}\left(\partial_{z} \eta(x)\right)^{2}+\int_{-\pi}^{\pi} \mathrm{d} \varphi\left[\eta(x)^{n-1} \partial_{z} \eta(x)\right]_{-\infty}^{\infty} \\
& =-(n-1) \int_{-\pi}^{\pi} \mathrm{d} \varphi \int_{-\infty}^{\infty} \mathrm{d} z \eta(x)^{n-2}\left(\partial_{z} \eta(x)\right)^{2},
\end{aligned}
$$


if $\eta(\varphi, z)$ or $\partial_{z} \eta(\varphi, z)$ vanish as $|z| \rightarrow \infty$. A similar procedure can be done for the term proportional to $D_{1}^{(n)}$, in the case that $\eta(\varphi, z)$ and $\partial_{\varphi} \eta(\varphi, z)$ are periodic functions in $\varphi$, with period $2 \pi$. Doing the same with the term proportional to $E_{1}^{(n)}$, we arrive to the desired expression for the order 2 of $F[\psi]$ in derivatives of $\eta$ :

$$
\begin{aligned}
F_{2}[\psi]= & \int_{x}\left\{Z_{1}(\psi(x))\left(\partial_{z} \psi\right)^{2}+Z_{2}(\psi(x))\left(\partial_{\varphi} \psi\right)^{2}\right. \\
& \left.+Z_{12}(\psi(x))\left(\partial_{\varphi} \psi\right)\left(\partial_{z} \psi\right)\right\},
\end{aligned}
$$

where the functions $Z_{1}(b+d), Z_{2}(b+d)$ and $Z_{12}(b+d)$ are defined as:

$$
\begin{aligned}
& Z_{1}(b+d)=\sum_{n \geq 2} \frac{n(n-1)}{(2 \pi)^{2}}\left[C_{1}^{(n)}-C_{2}^{(n)}\right] d^{n-2}, \\
& Z_{2}(b+d)=\sum_{n \geq 2} \frac{n(n-1)}{(2 \pi)^{2}}\left[D_{1}^{(n)}-D_{2}^{(n)}\right] d^{n-2}, \\
& Z_{12}(b+d)=\sum_{n \geq 2} \frac{n(n-1)}{(2 \pi)^{2}}\left[E_{1}^{(n)}-E_{2}^{(n)}\right] d^{n-2} .
\end{aligned}
$$

To calculate these functions in a simpler way, we may evaluate them for $d=0$, which gives us their value in $b$ :

$$
\begin{aligned}
& Z_{1}(b)=\frac{1}{2 \pi^{2}}\left[C_{1}^{(2)}(b)-C_{2}^{(2)}(b)\right], \\
& Z_{2}(b)=\frac{1}{2 \pi^{2}}\left[D_{1}^{(2)}(b)-D_{2}^{(2)}(b)\right], \\
& Z_{12}(b)=\frac{1}{2 \pi^{2}}\left[E_{1}^{(2)}(b)-E_{2}^{(2)}(b)\right] .
\end{aligned}
$$

Finally, to obtain the order two of $F[\psi]$, it is enough to change $b$ for $\psi(x)$ in the argument of these functions, and to replace them in Eq. (A21).

Thus, the second order DE is

$$
\begin{aligned}
F[\psi]= & \int_{x} b \mathcal{F}_{0}(b+\eta(x)) \\
& +\int_{x}\left\{Z_{1}(\psi(x))\left(\partial_{z} \psi\right)^{2}+Z_{2}(\psi(x))\left(\partial_{\varphi} \psi\right)^{2}\right. \\
& \left.+Z_{12}(\psi(x))\left(\partial_{\varphi} \psi\right)\left(\partial_{z} \psi\right)\right\} .
\end{aligned}
$$

We recall that we have assumed $F[\psi]$ to be analytic (as a functional) in a neighbourhood of $\psi \equiv a$ as well as the form factors $h^{(n)}$ at zero momenta. On the other hand, $\eta$ and $\partial_{z} \eta$ must tend to 0 as $|z| \rightarrow \infty$. Finally, $\psi$ and $\partial_{\varphi} \psi$ are periodic functions of $\varphi$, with period $2 \pi$. Except for the last condition, the other are equivalent to those required to apply the DE in Cartesian coordinates [11]. In addition, the method provides a tool to calculate the following orders, namely, by including higher order products of $k_{i}$ and $m_{i}$ in the expansion (A13).

Note that, from (A28), the order 2 we obtain is not proportional to the square of the gradient of $\psi$, as it happened in Cartesian coordinates. This is because, when considering the interaction energy between two planes $x_{3}=0$ and $x_{3}=\psi\left(x_{1}, x_{2}\right)$ in an isotropic space, the functional $F[\psi]$ must be invariant under rotations in the argument of $\psi$, this is, if $\left(x_{1}, x_{2}\right)^{T}$ is replaced by $R .\left(x_{1}, x_{2}\right)^{T}$, with $R \in S O(2)$. This symmetry is however lost when considering functions $\psi(\varphi, z)$, which justifies the mixed term in (A28). In the presence of extra symmetries (which may even be discrete), one could of course say more about the vanishing of one or more terms in DE.
[1] P. W. Milonni, The Quantum Vacuum (Academic Press, San Diego, 1994); K. A. Milton, The Casimir effect: Physical manifestations of zero-point energy (World Scientific, River Edge, USA, 2001; M. Bordag, G. L. Klimchitskaya, U. Mohideen, and V. M. Mostepanenko, Advances in the Casimir Effect (Oxford University Press, New York, 2009).

[2] C. Ccapa Ttira, C. D. Fosco, and E. L. Losada, J. Phys. A 43, 235402 (2010).

[3] B. V. Derjaguin, Koll. Z. 69, 155 (1934); B. V. Derjaguin and I. I. Abrikosova, Sov. Phys. JETP 3, 819 (1957); B. V. Derjaguin, Sci. Am. 203, 47 (1960); J. N. Israelachvili, Intermolecular and Surface Forces (Academic Press, London, 1992).
[4] J. Blocki, J. Randrup, W. J. Swiatecki, and C. F. Tsang, Ann. Phys. (Berlin) 105, 427 (1977).

[5] J. Blocki and W. J. Swiatecki, Ann. Phys. (Berlin) 132, 53 (1981).

[6] C. D. Fosco, F. C. Lombardo, and F. D. Mazzitelli, Phys. Rev. D 84, 105031 (2011).

[7] G. Bimonte, T. Emig, R. L. Jaffe, and M. Kardar, Europhys. Lett. 97, 50001 (2012).

[8] G. Bimonte, T. Emig, and M. Kardar, Appl. Phys. Lett. 100, 074110 (2012).

[9] C. D. Fosco, F. C. Lombardo, and F. D. Mazzitelli, Phys. Rev. D 85, 125037 (2012).

[10] C. D. Fosco, F. C. Lombardo, and F. D. Mazzitelli, Phys. Rev. D 86, 045021 (2012). 
[11] C. D. Fosco, F. C. Lombardo, and F. D. Mazzitelli, Phys. Rev. A 89, 062120 (2014).

[12] F. C. Lombardo, F. D. Mazzitelli, and P. I. Villar, J. Phys. A 41, 164009 (2008).

[13] I. Cavero-Peláez, K. A. Milton, P. Parashar, and K. V. Shajesh, Phys. Rev. D 78, 065019 (2008).

[14] J. Zinn-Justin, Quantum Field Theory and Critical Phenomena, International Series of Monographs on Physics Vol. 113 (Clarendon, Gloucestershire, UK, 2002), p. 1.
[15] D. A. R. Dalvit, F. C. Lombardo, F. D. Mazzitelli, and R. Onofrio, Phys. Rev. A 74, 020101 (2006).

[16] G. N. Watson, A Treatise on the Theory of Bessel Functions (Cambridge University Press, Cambridge, England, 1995).

[17] M. Abramowitz and I. Stegun, Handbook of Mathematical Functions, With Formulas, Graphs, and Mathematical Tables (Dover Publications, New York, 1974). 